\title{
Interpretation of extinction in Gaussian-beam scattering
}

\author{
NASA-CR-204823 \\ James A. Lock \\ Department of Physics, Clevelond State University, Cleveland, Ohio 44115
}

\begin{abstract}
Received July 22, 1994; revised manuscript received November 14, 1994; accepted November 15, 1994
The extinction efficiency for the interaction of a plane wave with a large nonabsorbing spherical particle is approximately 2.0. When a Gaussian beam of half-width $w_{0}$ is incident upon a spherical particle of radius $a$ with $w_{0} / a<1$, the extinction efficiency attains unexpectedly high or low values, contrary to intuitive expectations. The reason for this is associated with the so-called compensating term in the scattered field, which cancels the field of the Gaussian beam behind the particle, thereby producing the particle's shadow. I introduce a decomposition of the total exterior field into incoming and outgoing portions that are free of compensating terms. It is then shown that a suitably defined interaction efficiency has the intuitively expected asymptotic values of 2.0 for $w_{0} / a \gg 1$ and 1.0 for $w_{0} / a \ll 1$.
\end{abstract}

\section{INTRODUCTION}

In Lorenz-Mie theory, extinction is the name given to the energy lost by a plane wave during its interaction with a single spherical particle. ${ }^{1,2}$ The energy lost is said to be either absorbed or scattered. The absorbed energy excites the internal structure of the particle. The scattered energy is carried away from the particle by the radially outgoing electromagnetic waves created by the interaction. The scattered waves are further subdivided into diffracted waves created by the interaction of the plane wave with the geometry of the particle (i.e., the shape of its projected area) and specularly reflected and transmitted waves created by the interaction with the particle's composition (i.e., the strength of the interaction is determined by the particle's refractive index). If a plane wave is incident instead upon an ensemble of randomly positioned particles, then, in the single-scattering approximation, extinction describes the exponential attenuation of the undeflected portion of the plane wave as it passes through the ensemble. ${ }^{3-5}$

The purpose of this paper is to determine the physical interpretation of extinction if a Gaussian beam of halfwidth $w_{0}$ is incident upon a single spherical particle of radius $a$. If the particle is nonabsorbing, we intuitively expect that the extinction efficiency $\epsilon_{\text {ext }}$ (i.e., the extinction divided by the incident energy striking the surface of the particle) should behave in the following way as the width of the beam is varied. For plane-wave incidence and in the large-particle limit $a \gg \lambda$ the extinction effciency is approximately ${ }^{6.7} 2.0$. Half of this value is due to deflection of the geometrical rays that strike the particle's surface, and half is due to diffraction of the rays that graze its edge. ${ }^{B}$ For a narrow beam incident upon a large particle with $\omega_{0} \ll a$ the geometrical rays that strike the particle's surface are again deflected. But since the portion of the beam that grazes the particle's edge is exceedingly weak, diffraction is correspondingly weakened. Thus the extinction efficiency should approach 1.0 , being due solely to the deflected rays. When the extinction efficiency is computed for $w_{0} \ll a$, this expected reduction that is due to the weakening of diffraction does not occur. Rather, the extinction efficiency as a function of the particle size parameter continues to oscillate about 2.0. But the amplitude of the oscillations increases dramatically as $w_{0} / a$ decreases.

The first goal of this paper is to help us to understand physically what extinction describes for Gaussian-beam scattering and why the amplitude of the oscillations in $\epsilon_{\text {ext }}$ increases as $w_{0} / a$ decreases. The second goal is to construct another efficiency that has the intuitive limits of 2.0 for $w_{0} \gg a$ and 1.0 for $w_{0} \ll a$. Our pursuit of these goals proceeds in the following way. In Section 2 we briefly review the derivation of the extinction efficiency for a focused Gaussian beam striking a spherical particle head on. We then compute the extinction efficiency and observe its behavior as a function of the particle size parameter for various values of $w_{0} / a$. In Section 3 we show that this behavior results from associating extinction with the decomposition of the total electromagnetic fields exterior to the particle into a sum of two parts, the focused Gaussian beam in the absence of the target particle plus the scattered field produced by the target's presence. This decomposition is a natural choice when the scattered field only weakly perturbs the beam for $w_{0} / a \gg 1$. But when $w_{0} / a<1$ and the particle blocks off a large portion of the beam, the scattered field is no longer a weak perturbation, and this decomposition leads to certain difficulties in interpretation. In Section 4 we make an alternative decomposition of the electromagnetic fields into a sum of two other parts, a radially incoming part and a radially outgoing part. We define the interaction efficiency to be the energy carried by that portion of the outgoing wave that is distinguishable from the undeflected portion of the beam, divided by the incoming energy that strikes the particle's surface. We show that the interaction efficiency behaves in a way consistent with our intuition in both the $w_{0} / a \gg 1$ and the $w_{0} / a \ll 1$ limits.

\section{EXTINCTION EFFICIENCY FOR GAUSSIAN-BEAM SCATTERING}

\section{A. Beam When No Target Particle Is Present}

Consider a focused Gaussian beam propagating along the

$z$ axis of a coordinate system. This is called an on-axis 
beam. The time dependence of its electric and magnetic fields is $\exp (-i \omega t)$, which will hereafter be omitted. The beam has wavelength $\lambda$. It is focused by a lens to the half-width $w_{0}$ at the origin. One description of the electric and magnetic fields of the beam is given by the Davis first-order approximation ${ }^{9}$

$$
\begin{aligned}
\mathbf{E}_{\text {beam }}= & \frac{E_{0} \exp (i k z)}{1+2 i s z / w_{0}} \exp \left[\frac{-\left(x^{2}+y^{2}\right) / w_{0}^{2}}{1+2 i s z / w_{0}}\right] \\
& \times\left(\hat{u}_{x}-\frac{2 i s x / w_{0}}{1+2 i s z / w_{0}} \hat{u}_{z}\right), \\
\mathbf{B}_{\text {beam }}= & \frac{\left(E_{0} / c\right) \exp (i k z)}{1+2 i s z / w_{0}} \exp \left[\frac{-\left(x^{2}+y^{2}\right) / w_{0}^{2}}{1+2 i s z / w_{0}}\right] \\
& \times\left(\hat{u}_{y}-\frac{2 i s y / w_{0}}{1+2 i s z / w_{0}} \hat{u}_{z}\right),
\end{aligned}
$$

where the wave number is

$$
k=\frac{2 \pi}{\lambda}
$$

and the beam confinement parameter is

$$
s=\frac{1}{k w_{0}} .
$$

The plane-wave limit is obtained by setting $w_{0}-x$ and $s=0$ in Eqs. (1).

The beam fields have the partial-wave expansion ${ }^{10}$

$$
\begin{aligned}
\mathbf{E}_{\text {beam }}= & -i E_{0}(\cos \phi) \hat{u}_{r} \sum_{l=1}^{x} i^{l}(2 l+1) g_{l} \frac{j_{l}(k r)}{k r}(\sin \theta) \pi_{l}(\theta) \\
& +E_{0}(\cos \phi) \hat{u}_{\theta} \sum_{l=1}^{x} i^{l} \frac{2 l+1}{l(l+1)} g_{l}\left\{j_{l}(k r) \pi_{l}(\theta)\right. \\
& \left.-i\left[j_{l-1}(k r)-\frac{l}{k r} j_{l}(k r)\right] \tau_{l}(\theta)\right\} \\
& -E_{0}(\sin \phi) \hat{u}_{\phi} \sum_{l=1}^{x} i^{l} \frac{2 l+1}{l(l+1)} g_{l}\left\{j_{l}(k r) \pi_{l}(\theta)\right. \\
& \left.-i\left[j_{l-1}(k r)-\frac{l}{k r} j_{l}(k r)\right] \pi_{l}(\theta)\right\} \\
\mathbf{B}_{\text {beam }}= & \frac{-i E_{0}}{c}(\sin \phi) \hat{u}_{r} \sum_{l=1}^{x} i^{l}(2 l+1) g_{l} \frac{j_{l}(k r)}{k r}(\sin \theta) \pi_{l}(\theta) \\
& +\frac{E_{0}}{c}(\sin \phi) \hat{u}_{\theta} \sum_{l=l}^{x} i^{l} \frac{2 l+1}{l(l+1)} g_{l}\left\{j_{l}(k r) \pi_{l}(\theta)\right. \\
& \left.-i\left[j_{l-1}(k r)-\frac{l}{k r} j_{l}(k r)\right] \tau_{l}(\theta)\right\} \\
& +\frac{E_{0}}{c}(\cos \phi) \hat{u}_{\phi} \sum_{l=1}^{\infty} i^{l} \frac{2 l+1}{l(l+1)} g_{l}\left\{j_{l}(k r) \tau_{l}(\theta)\right. \\
& \left.-\left[j_{l-1}(k r)-\frac{l}{k r} j_{l}(k r)\right] \pi_{l}(\theta)\right\} .
\end{aligned}
$$

In these expressions the $j_{l}(k r)$ are spherical Bessel functions, and the angular functions $\pi_{l}(\theta)$ and $\tau_{l}(\theta)$ are related to associated Legendre polynomials by

$$
\pi_{l}(\theta)=\frac{P_{l}^{1}(\cos \theta)}{\sin \theta}, \quad \tau_{l}(\theta)=\frac{\mathrm{d}}{\mathrm{d} \theta} P_{l}^{1}(\cos \theta) .
$$

The shape of the beam is determined by the coefficients $g_{l}$, which are weighting factors for the individual partial waves. Alternatively, these beam shape coefficients may be obtained from the beam profiles by ${ }^{11}$

$$
\begin{aligned}
g_{l}= & \frac{(-i)^{l-1}}{2} \frac{k r}{j_{l}(k r)} \frac{1}{l(l+1)} \int_{0}^{\pi} \sin ^{2} \theta \mathrm{d} \theta f(k r, \theta) \\
& \times \exp (i k r \cos \theta) P_{l}{ }^{1}(\cos \theta),
\end{aligned}
$$

where the radial components of the electric and magnetic fields of the on-axis beam assume the forms

$$
\begin{aligned}
E_{\text {beam }}^{\text {radial }} & =E_{\text {beam }}^{x} \sin \theta \cos \phi+E_{\text {beam }}^{z} \cos \theta \\
& =E_{0} \exp (i k r \cos \theta) f(k r, \theta) \sin \theta \cos \phi, \\
B_{\text {bead }}^{\text {radial }} & =B_{\text {beam }}^{y} \sin \theta \sin \phi+B_{\text {beam }}^{z} \cos \theta \\
& =\frac{E_{0}}{c} \exp (i k r \cos \theta) f(k r, \theta) \sin \theta \sin \phi .
\end{aligned}
$$

For an on-axis Gaussian beam focused at the origin the beam shape coefficients are approximated to a high degree of accuracy ${ }^{11}$ by the localized beam model ${ }^{12}$

$$
g_{l} \approx \exp \left[-s^{2}(l+1 / 2)^{2}\right]
$$

The partial-wave expansion of a plane wave is obtained in the limit $s=0$ and $g_{l}=1$.

\section{B. Scattered Wave Produced by the}

Beam-Particle Interaction

Consider now that the Gaussian beam approximated by Eqs. (1) or relation (8) substituted into Eqs. (4) is incident upon a spherical particle of radius $a$ and refractive index $n$ whose center is at the origin of coordinates. The standard method for solving for this electromagnetic boundary-value problem begins with the decomposition of the electric and magnetic fields exterior to the particle into a sum of two parts ${ }^{13-15}$ :

$\mathbf{E}_{\text {total }}=\mathbf{E}_{\text {beam }}+\mathbf{E}_{\text {scattered }}, \quad \mathbf{B}_{\text {total }}=\mathbf{B}_{\text {beam }}+\mathbf{B}_{\text {scattered }}$.

The fields $\mathbf{E}_{\text {beam }}$ and $\mathbf{B}_{\text {beam }}$ are the electromagnetic fields of the beam in the absence of the particle as given in Eqs. (4), and $\mathbf{E}_{\text {scatcered }}$ and $\mathbf{B}_{\text {scatcered }}$ represent the change in the total fields that is due to placing the target particle in the beam's path. Specifically, the decomposition of Eqs. (9) gives $^{10}$

$$
\begin{aligned}
\mathbf{E}_{\text {scattered }}= & i E_{0}(\cos \phi) \hat{u}_{r} \sum_{l=1}^{x} i^{l}(2 l \div 1) g_{l} a_{l} \frac{h_{l}^{(1)}(k r)}{k r}(\sin \theta) \\
& \times \pi_{l}(\theta)-E_{0}(\cos \phi) \hat{u}_{\theta} \sum_{l=1}^{x} i^{l} \frac{2 l+1}{l(l+1)} \\
& \times g_{l}\left\{b_{l} h_{l}^{(1)}(k r) \pi_{l}(\theta)-i a_{l}\left[h_{l-l}^{(1)}(k r)\right.\right. \\
& \left.\left.-\frac{l}{k r} h_{l}^{(1)}(k r)\right] \tau_{l}(\theta)\right\}+E_{0}(\sin \phi) \hat{u}_{\phi} \\
& \times \sum_{l=1}^{x} i^{l} \frac{2 l+1}{l(l+1)} g_{l}\left\{b_{l} h_{l}^{(1)}(k r) \tau_{l}(\theta)\right. \\
& \left.-i a_{l}\left[h_{l-1}^{(1)}(k r)-\frac{l}{k r} h_{l}^{(1)}(k r)\right] \pi_{l}(\theta)\right\}
\end{aligned}
$$




$$
\begin{aligned}
\mathbf{B}_{\text {seattered }}= & \frac{i E_{0}}{c}(\sin \phi) \tilde{u}_{r} \sum_{l=1}^{\infty} i^{l}(2 l+1) g_{l} b_{l} \frac{h_{l}^{(1)}(k r)}{k r}(\sin \theta) \\
& \times \pi_{l}(\theta)-\frac{E_{0}}{c}(\sin \phi) \dot{u}_{\theta} \sum_{l=1}^{\infty} i^{l} \frac{2 l+1}{l(l+1)} \\
& \times g_{l}\left\{a_{l} h_{l}^{(1)}(k r) \pi_{l}(\theta)-i b_{l}\left[h_{l-1}^{(1)}(k r)\right.\right. \\
& \left.\left.-\frac{l}{k r} h_{l}^{(1)}(k r)\right] \tau_{l}(\theta)\right\}-\frac{E_{0}}{c}(\cos \phi) \hat{u}_{\phi} \sum_{l=1}^{\infty} i^{l} \\
& \times \frac{2 l+1}{l(l+1)} g_{l}\left\{a_{l} h_{l}^{(1)}(k r) \tau_{l}(\theta)\right. \\
& \left.-i b_{l}\left[h_{l-1}^{(1)}(k r)-\frac{l}{k r} h_{l}^{(1)}(k r)\right] \pi_{l}(\theta)\right\}, \quad(10)
\end{aligned}
$$

where the $h_{l}^{(1)}(k r)$ are spherical Hankel functions of the first type and $a_{l}$ and $b_{l}$ are the partial-wave scattering amplitudes of plane-wave Lorenz-Mie theory.

The total exterior field is illustrated in Fig. 1 for the case in which the beam is wide and the particle is small. At most locations in space the total fields strongly resemble the original beam, $\mathbf{E}_{\text {scattered }}$ and $\mathbf{B}_{\text {scattered }}$ represent only a small perturbation, and the decomposition of Eqs. (9) makes good physical sense. An exception, however, is provided by the deep-shadow region immediately behind the particle, where the particle blocks off the original beam. ${ }^{16,17}$ The deep-shadow region is denoted by $\mathrm{S}$ in Fig. 1. In spite of the particle's blocking off the incident beam in the deep-shadow region, the total field there is not necessarily small if the particle is transparent. In particular, surface waves associated with reflection pass through this region (see Fig. 46 of Ref. 8), and the transmitted waves form a spherical aberration caustic there. The term deep-shadow region refers instead to the fact that the waves diffracted by the particle only gradually spread to fill the space behind it (see Fig. 14 of Ref. 17). Similarly, the term block off means that, although the fields in the deep-shadow region are nonzero, they do not at all resemble the incident beam. The incident beam has been totally removed and replaced by the reflection surface waves and transmitted waves. As a result, in the deep-shadow region the scattered fields must assume the form ${ }^{16}$

$$
\begin{aligned}
& \mathbf{E}_{\text {scattered }}=\mathbf{E}_{\text {compensating }}+\mathbf{E}_{\text {residual }}, \\
& \mathbf{B}_{\text {scattered }}=\mathbf{B}_{\text {compensating }}+\mathbf{B}_{\text {ressidual }},
\end{aligned}
$$

where

$$
\mathbf{E}_{\text {compense ting }}=-\mathbf{E}_{\text {beam }}, \quad \mathbf{B}_{\text {compenatiog }}=-\mathbf{B}_{\text {beem }} .
$$

The compensating term in the scattered field cancels the beam field and thereby mathematically ensures its removal from the deep-shadow region. The residual fields are the transmitted waves and the reflection surface waves present in the deep-shadow region if the particle is transparent. If it is opaque, the residual fields are only the reflection surface waves. ${ }^{17}$

\section{Extinction Efficiency}

Using the decomposition of Eqs. (9), we can define the absorption, scattering, and extinction cross sections as ${ }^{2.10 .18}$

$$
\begin{aligned}
C_{\text {absorption }}= & \frac{-c}{E_{0}^{2}} \int_{0}^{\pi} \sin \theta \mathrm{d} \theta \int_{0}^{2 \pi} \mathrm{d} \phi \operatorname{Re}\left(\mathbf{E}_{\text {tolal }}^{*} \times \mathbf{B}_{\text {total }}\right) \\
C_{\text {xcattering }}= & \frac{c}{E_{0}^{2}} \int_{0}^{\pi} \sin \theta \mathrm{d} \theta \int_{0}^{2 \pi} \mathrm{d} \phi \\
& \times \operatorname{Re}\left(\mathbf{E}_{\text {cattered }}^{*} \times \mathrm{B}_{\text {scattered }}\right) \\
= & \frac{2 \pi}{k^{2}} \sum_{l=1}^{\pi}(2 l+1)\left|g_{l}\right|^{2}\left(\left|a_{l}\right|^{2}+\left|b_{l}\right|^{2}\right), \\
C_{\text {extinction }}= & \frac{-c}{E_{0}^{2}} \int_{0}^{\pi} \sin \theta \mathrm{d} \theta \int_{0}^{2 \pi} \mathrm{d} \phi \\
& \times \operatorname{Re}\left(\mathbf{E}_{\text {besm }}^{*} \times \mathrm{B}_{\text {scattered }}+\mathbf{E}_{\text {scattered }}^{*} \times \mathbf{B}_{\text {beam }}\right) \\
= & \frac{2 \pi}{k^{2}} \sum_{l=1}^{\pi}(2 l+1)\left|g_{l}\right|^{2} \operatorname{Re}\left(a_{l}+b_{l}\right),
\end{aligned}
$$

respectively. They reduce to the usual Lorenz-Mie expressions in the plane-wave limit $g_{l}=1$. Since the bearn's angle-integrated Poynting vector vanishes, i.e.,

$$
\frac{c}{E_{0}^{2}} \int_{0}^{\pi} \sin \theta \mathrm{d} \theta \int_{0}^{2 \pi} \mathrm{d} \phi \operatorname{Re}\left(E_{\text {beam }}^{*} \times B_{\text {beam }}\right)=0
$$

the absorption, scattering, and extinction cross sections are related to one another by

$$
C_{\text {extinction }}=C_{\text {scatterng }}+C_{\text {absorption }}
$$

Equation (17) describes conservation of energy for the beam-particle system. The absorption, scattering. and extinction efficiencies are obtained by dividing

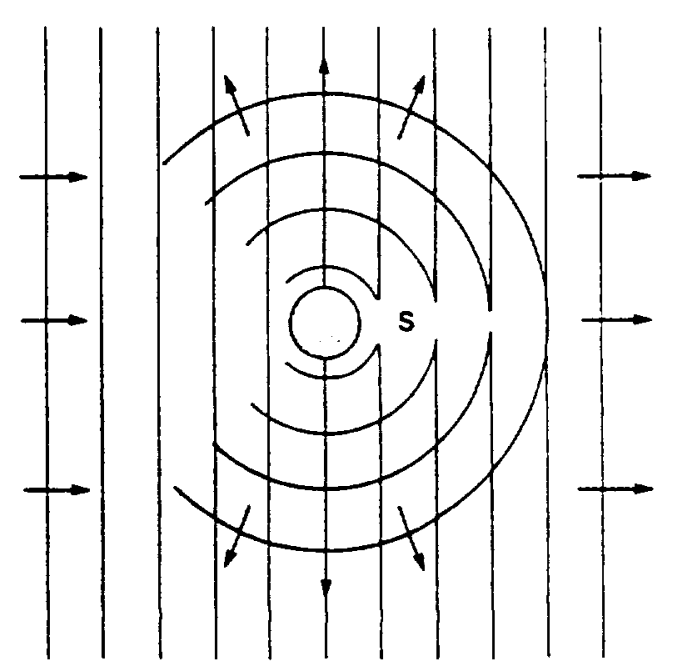

Fig. 1. Scattering of a plane wave by a spherical particle The small region $S$ to the right of the particle denotes the deep-shadow region, where the plane wave has been removed by the particle in the near-forward direction and in the near zone and replaced by the transmitted wave and the reflection surface waves. 
Eqs. (13)-(15) by the cross section for radiation striking the particle surface,

$$
\begin{aligned}
C_{\text {incident }}= & -\frac{c}{E_{0}^{2}} \int_{\pi / 2}^{\pi} \sin \theta \mathrm{d} \theta \int_{0}^{2 \pi} \mathrm{d} \phi \\
& \times\left[\operatorname{Re}\left(\mathbf{E}_{\text {benm }}^{*} \times \mathrm{B}_{\text {beam }}\right) \cdot \hat{u}_{r}\right]_{r=a},
\end{aligned}
$$

giving, for example,

$$
\epsilon_{\text {extinction }}=\frac{C_{\text {extinetion }}}{C_{\text {incident }}} .
$$

In the Davis first-order beam approximation the incident cross section is

$$
\begin{aligned}
C_{\text {incident }}= & 2 \pi a^{2}\left(1+\frac{4 s^{2} a^{2}}{w_{0}^{2}}\right) \\
& \times \int_{0}^{1} u \mathrm{~d} u \frac{\exp \left[\frac{-2 a^{2}\left(1-u^{2}\right) / w_{0}^{2}}{1+4 s^{2} a^{2} u^{2} / w_{0}^{2}}\right]}{\left(1+4 s^{2} a^{2} u^{2} / w_{0}^{2}\right)^{2}} .
\end{aligned}
$$

In the plane-wave limit $s \rightarrow 0$, Eq. (20) reduces to $C_{\text {incident }}=\pi a^{2}$.

The behavior of the extinction efficiency of Eq. (19) as the width of the beam is varied is illustrated in the following example. Consider a spherical water droplet of radius $a=50 \mu \mathrm{m}$ and $n=1.333$ illuminated on axis by a Gaussian beam focused at the origin with $0.57 \mu \mathrm{m} \leq \lambda \leq$ $0.64 \mu \mathrm{m}$ and $0.1 \leq w_{0} / a \leq 100$. These values of $w_{0}$ are well within the region of applicability of both the Davis first-order beam approximation of Eqs. (1) and the localized model for the beam shape coefficients of relation (8).

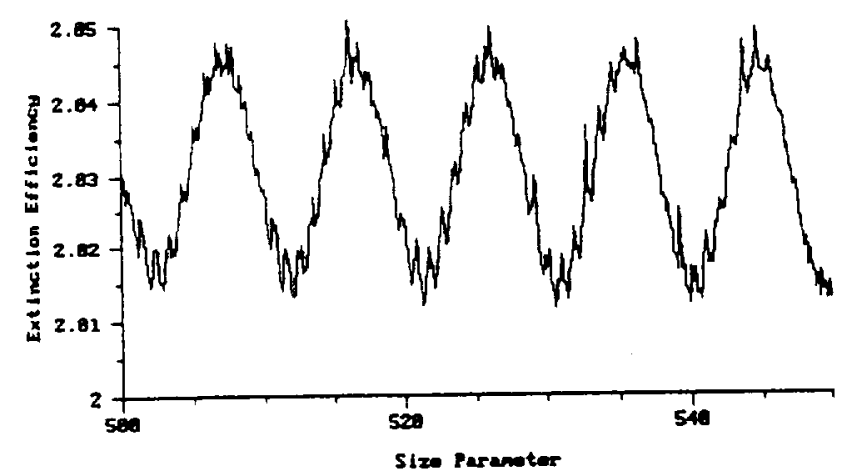

(a)

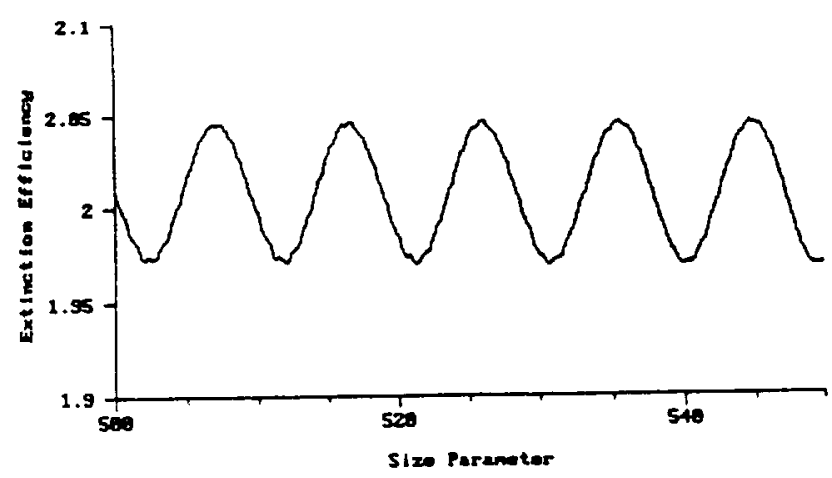

(b)
The incident cross section of Eq. (20) was computed by numerical integration with a 5001-point grid. The resulting extinction efficiency of Eq. (19) is shown as a function of the particle size parameter

$$
x=\frac{2 \pi a}{\lambda}
$$

for $w_{0} / a=20.0,1.0,0.2$, and 0.1 in Figs. 2(a), 2(b), 2(c), and 2(d), respectively, and as a function of $w_{0} / a$ for $\lambda=0.6328 \mu \mathrm{m}$ in Fig. 3. For $w_{0} / a \gg 1$ we find that $\epsilon_{\text {ext }}=2.0$, in agreement with the plane-wave limit. This result is interpreted as being an amount 1.0 that is due to scattering plus another 1.0 that is due to diffraction. ${ }^{6}$ In addition, the extinction efficiency as a function of size parameter possesses oscillations, known as the interference structure, that are due to interference between the diffracted and transmitted fields. ${ }^{19,20}$ The efficiency also exhibits the so-called ripple structure that is due to morphology-dependent resonances. ${ }^{21}$ For $w_{0} / a<0.5$ the extinction efficiency as a function of size parameter continues to oscillate about 2.0. But the amplitude of the interference structure increases dramatically. The ripple structure is now absent, since the Gaussian beam no longer extends out to the edges of the particle, where morphology-dependent resonances are most efficiently excited. ${ }^{22}$ The increase in the amplitude of the interference structure is contrary to our expectation that the diffraction contribution to the extinction efficiency should decrease because a progressively weaker portion of the beam grazes the edge of the particle. In Section 3 below we demonstrate that the large-amplitude interference structure for $w_{0} / a \ll 1$ results from the interference of the transmitted field with the compensating field.

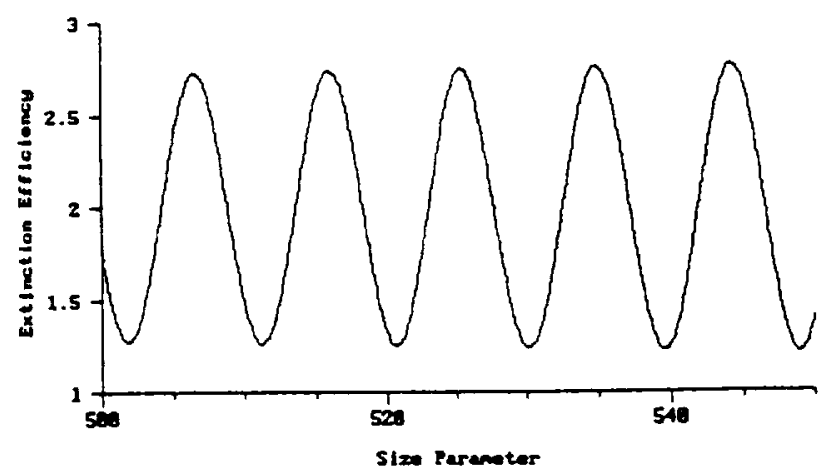

(c)

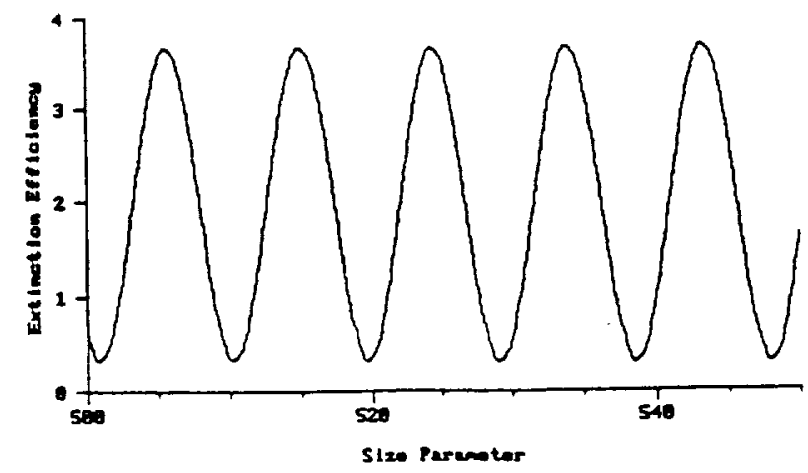

(d)

Fig. 2. Extinction efficiency of Eq. (19) as a function of the particle size parameter for (a) $k w_{0}=10^{4},(\mathrm{~b}) k w_{0}=500$, (c) $k w_{0}=100$, and (d) $k w_{0}=50$, corresponding to $w_{0} / a-20.0,1.0,0.2$, and 0.1 , respectively. 


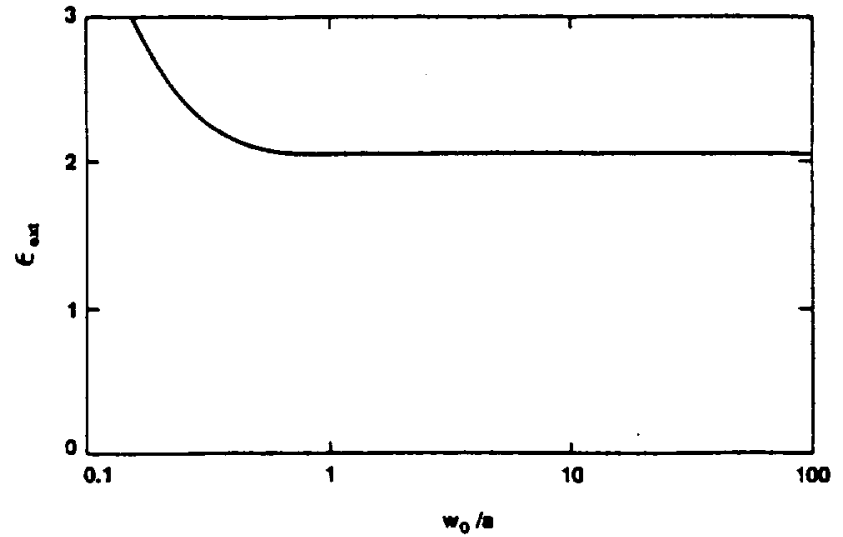

Fig. 3. Extinction efficiency of Eq. (19) as a function of the half-width of the beam divided by the particle radius for a beam wavelength of $\lambda=0.6328 \mu \mathrm{m}$, a particle radius of $a=50 \mu \mathrm{m}$, and a refractive index of $n=1.333$.

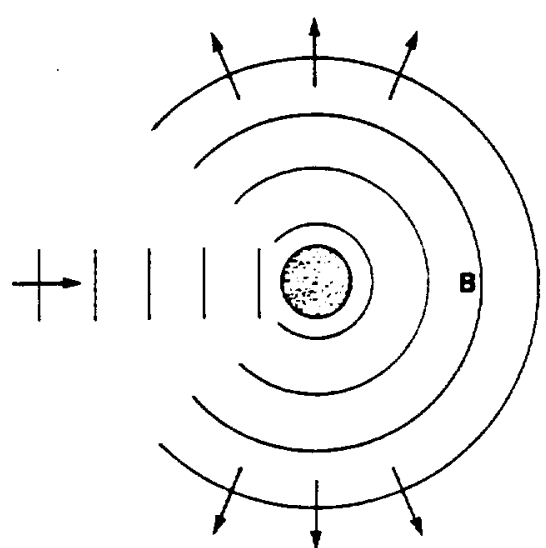

Fig. 4. Scattering of a narrow beam by a spherical particle. The region $B$ to the right of the particle and extending to the far zone denotes the region where the beam has been removed by the particle and replaced by the transmitted and reflected waves.

\section{COMPENSATING FIELD IN GAUSSIAN-BEAM SCATTERING}

Figure 4 illustrates the interaction of a narrow beam with a large particle. The particle blocks off most of the beam, preventing its continued propagation to $z-\infty$. Again the term block off means that the beam behind the particle has been removed and replaced by the transmitted and reflected waves. On the other hand, the decomposition of the total fields in Eqs. (9) contains $\mathbf{E}_{\text {beam }}$ and $\mathbf{B}_{\text {beam, }}$, which in fact are not present behind the particle. Thus $\mathbf{E}_{\text {scattered }}$ and $\mathbf{B}_{\text {scattered }}$ in the near-forward direction and in both the near zone and the far zone must be of the form of Eqs. (11) so that the compensating field can cancel the beam field and thereby ensure the beam's absence behind the particle. ${ }^{16}$

The compensating field is easily identified in numerical computations of the far-zone scattered intensity. Consider a focused on-axis Gaussian beam with $\omega_{0}=10 \mu \mathrm{m}$ and $\lambda=0.6204 \mu \mathrm{m}$ incident upon a spherical water droplet with $a=50 \mu \mathrm{m}$ and $n=1.333$. This wavelength is equivalent to the size parameter $x=506.38$ and corresponds to the first maximum of the extinction efficiency graph of Fig. 2(c), where $\epsilon_{\text {ext }}=2.734$. The far-zone scat- tered intensity

$$
I_{\text {scatlered }}(\theta, \phi) \hat{u}_{r}=\lim _{r \rightarrow \infty} \operatorname{Re}\left(\mathbf{E}_{\text {scatlered }}^{*} \times \mathbf{B}_{\text {scattered }}\right)
$$

is graphed in Fig. $5(a)$ for $-180^{\circ} \leq \theta \leq 180^{\circ}$ and $\phi=$ $90^{\circ}$. The scattering is dominated in the forward hemisphere by transmission and in the backward hemisphere by specular reflection and transmission following one internal reflection. ${ }^{11}$ Since $w_{0} / a \ll 1$, diffraction in the near-forward direction is minimal. In Fig. 5(a) there is a large peak at $-1.5^{\circ} \leq \theta \leq 1.5^{\circ}$ that is not observed in experiments. ${ }^{23.24}$ I claim that this peak is the intensity corresponding to the compensating field. The inset of Fig. 5(a) shows the scattered intensity for $0^{\circ} \leq \theta \leq 5^{\circ}$, illustrating the constructive interference between the compensating field $(C)$ and the transmitted field $(T)$ for $\lambda=$ $0.6204 \mu \mathrm{m}$. Since the scattered intensity for $-5^{\circ}<\theta<$ $5^{*}$ is orders of magnitude larger than at any other scattering angle, integrating over this constructive interference produces the increased extinction efficiency $\epsilon_{\text {ext }}=2.734$ shown in Fig. 2(c).

Similarly, the far-zone scattered intensity for $\lambda=$ $0.6261 \mu \mathrm{m}$ is shown in Fig. $5(\mathrm{~b})$. This wavelength is equivalent to $x=501.77$ and corresponds to the first minimum of the extinction efficiency graph of Fig. 2(c), where $\epsilon_{\text {ext }}=1.271$. The inset of Fig. 5(b) illustrates the destructive interference between the compensating field (C) and the transmitted field $(T)$ for $\lambda=0.6261 \mu \mathrm{m}$. Integrating over this destructive interference produces the decreased extinction efficiency of $\epsilon_{\text {ext }}=1.271$. A detailed physical model of the oscillations in the extinction efficiency as a function of size parameter for $w_{0} / a \ll 1$ based on compensating field-transmission field interference will be given elsewhere.

As interesting as these large-amplitude oscillations in $\epsilon_{\text {ext }}$ for $w_{0} / a \ll 1$ appear to be, they are not observable. Figures 5(c) and 5(d) graph the far-zone intensity associated with the total field exterior to the particle, ${ }^{25}$

$$
I_{\text {total }}(\theta, \phi) \hat{u}_{r}=\lim _{\sim \rightarrow \infty} \operatorname{Re}\left(\mathbf{E}_{\text {totsl }}^{*} \times \mathbf{B}_{\text {total }}\right) \text {, }
$$

for $-180^{\circ} \leq \theta \leq 180^{\circ}$ and $\phi=90^{\circ}$ for $\lambda=0.6204 \mu \mathrm{m}$ and $\lambda=0.6261 \mu \mathrm{m}$, respectively. Figures $5(\mathrm{c})$ and $5(\mathrm{~d})$ are identical to Figs. 5(a) and 5(b) except that the beam fields have canceled the compensating portion of the scattered fields and have eliminated the spurious peak in Figs. 5(a) and $5(\mathrm{~b})$. This cancellation verifies my earlier claim of having properly identified the compensating field peak in Figs. 5(a) and 5(b). It also affects the physical meaning of the extinction efficiency. Since the large-amplitude interference structure in $\epsilon_{\text {ext }}$ was produced by the interference between the compensating field and the transmitted field and since only the total field is observed in experiments, the compensating field-transmission field interference is not observable. If another efficiency based on the total field were to be constructed, the cancellation of the beam by the compensating field would prevent the large-amplitude interference structure in the other efficiency from occurring.

The compensating term in the scattered field in the near-forward direction for $w_{0} / a \leq 1$ can also be demonstrated analytically. For small $\theta$ the angular functions of Eqs. (5) are approximated by ${ }^{26}$

$$
\pi_{l}(\theta) \approx \tau_{l}(\theta)=\frac{l(l+1)}{2} J_{0}[(l+1 / 2) \theta] \text {. }
$$




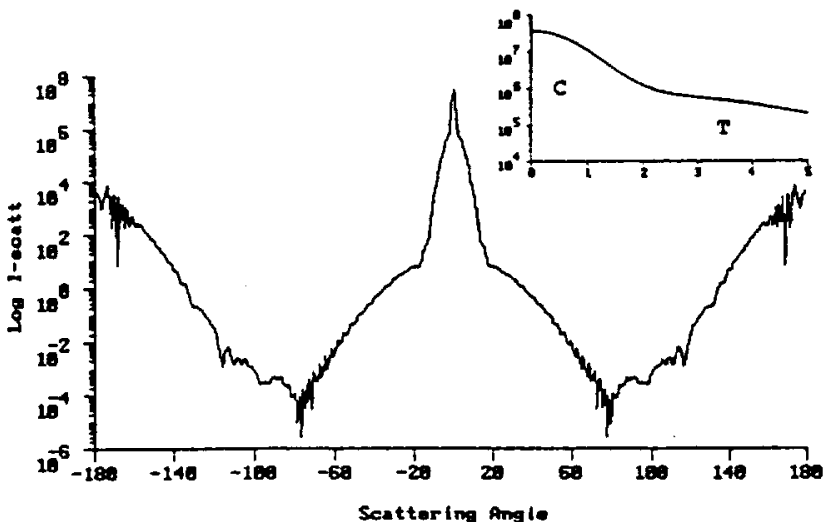

(a)

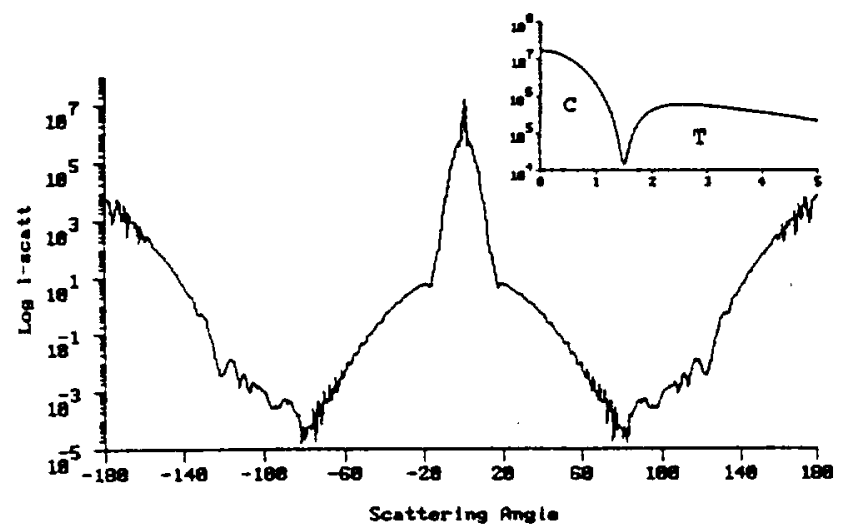

(b)

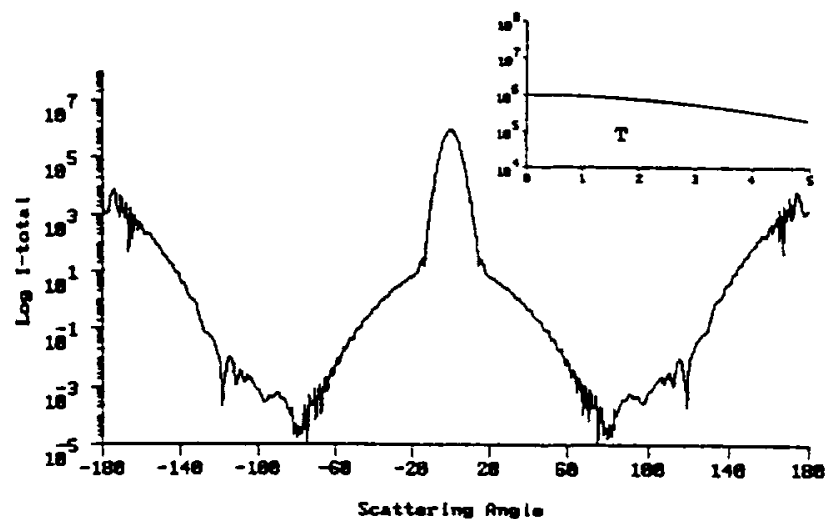

(c)

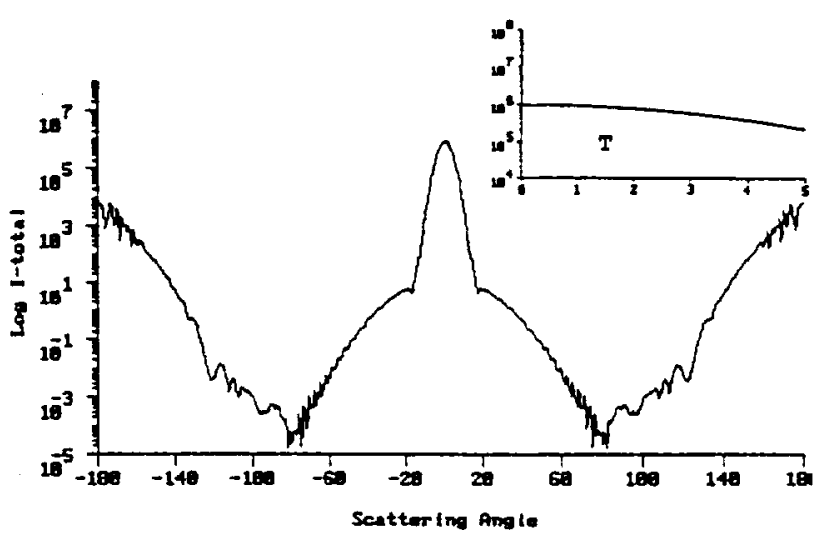

(d)

Fig. 5. Scattered intensity as a function of the scattering angle $\theta$ for $\phi=90^{\circ}$ for a beam of half-width $w_{0}=10 \mu \mathrm{m}$ and wavelength (a) $\lambda=0.6261 \mu \mathrm{m}$ and (b) $\lambda=0.6204 \mu \mathrm{m}$ incident upon a spherical particle of radius $a=50 \mu \mathrm{m}$ and refractive index $n=1.333$. The insets in (a) and (b) show the intensity for $0^{\circ} \leq \theta \leq 5^{\circ}$. The region $C$ is dominated by the compensating field, and the region $T$ is dominated by the transmitted field. Also shown is the total intensity as a function of the scattering angle $\theta$ for $\phi=90^{\circ}$ and the same beam and particle parameters for (c) $\lambda=0.6261 \mu \mathrm{m}$ and (d) $\lambda=0.6204 \mu \mathrm{m}$. The compensating field peak evident in (a) and (b) is now absent because of its cancellation by the beam field. The insets in (c) and (d) again show the intensity for $0^{\circ} \leq \theta \leq 5^{\circ}$, where transmission is the largest contribution to the total intensity.

The partial-wave expansion for the beam electric field in the far-zone limit $r \rightarrow x$ then becomes

$$
\mathbf{E}_{\text {benm }}=\frac{-i E_{0}}{k r} \exp (i k r) \hat{u}_{x} \sum_{l=1}^{x}(l+1 / 2) g_{l} J_{0}[(l+1 / 2) \theta]
$$

where we have used

$$
\begin{aligned}
\lim _{r \rightarrow \infty} j_{l}(k r) & =\frac{1}{k r} \sin \left(k r-\frac{l \pi}{2}\right), \\
\hat{u}_{x} & =(\cos \phi) \hat{u}_{\theta}-(\sin \phi) \hat{u}_{\phi}
\end{aligned}
$$

for $\theta=0^{\circ}$. Converting the sum over partial waves to an integral over an associated impact parameter $u$ in an approximate way and substituting relations (8), we then obtain ${ }^{27,28}$

$$
\begin{aligned}
\mathbf{E}_{\text {beam }} & =\frac{-i E_{0}}{k r} \exp (i k r) \hat{u}_{x} \int_{0}^{\infty} u \mathrm{~d} u \exp \left(-s^{2} u^{2}\right) J_{0}(u \theta) \\
& =\frac{-i E_{0}}{k r} \exp (i k r) \hat{u}_{x} \frac{1}{2 s^{2}} \exp \left(-\theta^{2} / 4 s^{2}\right)
\end{aligned}
$$

On the other hand, the scattered electric field of Eqs. (10) under the same assumptions of small $\theta$ and $r-x$ is

$$
\begin{aligned}
\mathbf{E}_{\text {scattered }}= & \frac{i E_{0}}{k r} \exp (i k r) \hat{u}_{z} \sum_{l=1}^{l_{\max }}(l+1 / 2) g_{l}\left(a_{l}+b_{l}\right) \\
& \times J_{0}[(l+1 / 2) \theta]
\end{aligned}
$$

where

$$
\begin{aligned}
\varliminf_{l} h_{l}^{(1)}(k r) & =\frac{(-i)^{l+1}}{k r} \exp (i k r), \\
l_{\max } & =2+k a+4.3(k a)^{1 / 3} .
\end{aligned}
$$

In the large-particle limit $k a \gg 1 \mathrm{Eq}$. (31) becomes approximately

$$
l_{\max } \approx k a \text {. }
$$

We now show that, for Gaussian-beam scattering, the compensating field of Eqs. (11) is contained in the portion of the partial-wave scattering amplitudes $a_{l}$ and $b_{l}$ that one usually associates with diffraction. The Debye-series 
expansion of the plane-wave Mie theory partial-wave scattering amplitudes is ${ }^{28.29}$

$$
\left.\begin{array}{l}
a_{l} \\
b_{l}
\end{array}\right\}=\frac{1}{2}\left[1-R_{l}^{22}-\sum_{p=1}^{x} T_{l}^{21}\left(R_{l}^{11}\right)^{p-1} T_{l}^{12}\right],
$$

where the first term (1) denotes diffraction, the second term $\left(-R_{l}^{22}\right)$ denotes specular reflection, and the third term $\left[-T_{l}^{21}\left(R_{l}^{11}\right)^{p-1} T_{l}^{12}\right]$ denotes transmission following $p-1$ internal reflections. The diffracted portion of the scattered field is then

$$
\mathbf{E}_{\text {scactered }}^{\text {diff }} \approx \frac{i E_{0}}{k r} \exp (i k r) \hat{u}_{x} \sum_{l=1}^{k a}(l+1 / 2) g_{l} J_{0}[(l+1 / 2) \theta] \text {. }
$$

Again converting the sum over partial waves to an integral over an associated impact parameter $u$, we obtain

$$
\mathbf{E}_{\text {scattered }}^{\mathrm{dif}}=\frac{i E_{0}}{k r} \exp (i k r) \hat{u}_{x} \int_{0}^{k a} u \mathrm{~d} u \exp \left(-s^{2} u^{2}\right) J_{0}(u \theta),
$$

where $\mathrm{w}^{30-32}$ general present at a given point in space, they are distinguishable because the wave fronts of the outgoing beam are flat and its amplitude is constant, whereas the wave fronts of the scattered wave are spherical and its amplitude falls as $1 / r$ (see Fig. 1). For a narrow beam incident upon a large particle with $w_{0} / \alpha \ll 1$ the beam and the scattered parts are not individually observable, since the particle has removed the beam in the near-forward direction and replaced it by the transmitted and reflected waves. The removal of the beam is not evidenced in either $\mathbf{E}_{\text {beam }}$ or $\mathbf{E}_{\text {scattered }}$ taken individually but is seen only when they are added together to form $\mathbf{E}_{\text {total }}$. In experiments, however, only the total field is observed. The extinction efficiency exhibits interference between the compensating and transmitted portions of the scattered field that cannot be observed because of the beam field-compensating field cancellation.

\section{ALTERNATTVE DECOMPOSITION OF THE TOTAL FIELD}

Since the spherical Bessel function $j_{l}(k r)$ in Eqs. (4) may be written as

$$
\int_{0}^{k a} u \mathrm{~d} u \exp \left(-s^{2} u^{2}\right) J_{0}(u \theta)=\left\{\begin{array}{ll}
\frac{1}{2 s^{2}} \exp \left(-a^{2} / w_{0}^{2}\right) \sum_{n=1}^{x}\left(\frac{2 a^{2}}{w_{0}^{2}}\right)^{n} \frac{J_{n}(k a \theta)}{(k a \theta)^{n}}, & w_{0} \geq a \\
\frac{1}{2 s^{2}} \exp \left(-\theta^{2} / 4 s^{2}\right)-\frac{1}{2 s^{2}} \exp \left(-a^{2} / w_{0}^{2}\right) \sum_{n=0}^{x}\left(\frac{-w_{0}^{2}}{2 a^{2}}\right)^{n}(k a \theta)^{n} J_{n}(k a \theta), & w_{0} \leq a
\end{array} .\right.
$$

For a wide beam incident upon a small particle with $w_{0} / a \gg 1$ the diffracted electric field is approximately

$$
\mathbf{E}_{\text {scattered }}^{\text {diff }}=\frac{i E_{0}}{k r} \exp (i k r) \hat{u}_{x} \exp \left(-a^{2} / w_{0}^{2}\right)(k a)^{2} \frac{J_{1}(k a \theta)}{k a \theta}
$$

This is the usual state of affairs in plane-wave Mie theory. But for $w_{0} \ll a$ a comparison of relation (28) with relation (35) and Eq. (36) leads us to identify the first term in the second line of Eq. (36) substituted into relation (35) as the compensating field. After the cancellation of the compensating field and the beam field the remaining part of $\mathbf{E}_{\text {seatuered, given by }}^{\text {dif }}$

$$
\begin{aligned}
\mathbf{E}_{\text {scattered }}^{\text {diff. remainder }}= & \frac{-i E_{0}}{k r} \exp (i k r) \hat{u}_{x} \frac{1}{2 s^{2}} \exp \left(-a^{2} / w_{0}{ }^{2}\right) \\
& \times J_{0}(k a \theta)
\end{aligned}
$$

describes the weak diffraction produced by the tail of the beam grazing the edge of the particle. A qualitative and intuitive derivation of relation (38) is given in Appendix A below.

It is now clear what extinction in Gaussian-beam scattering describes and why the extinction efficiency of Eq. (19) behaves in a counterintuitive way for $w_{0} / a<1$. Extinction is based on the decomposition of the total fields exterior to the particle into the sum of a beam part and a scattered part as in Eqs. (9) and on the implicit assumption that each part individually is observable. For a wide beam incident upon a small particle with $w_{0} / a \gg 1$ this is indeed the case. Although both the undeflected portion of the original beam and the scattered wave are in

$$
j_{l}(k r)=1 / 2 h_{l}^{(1)}(k r)+1 / 2 h_{l}^{(2)}(k r),
$$

where $h_{l}^{(1)}(k r)$ describes radially outgoing waves and $h_{l}^{(2)}(k r)$ describes radially incoming waves, ${ }^{33}$ by substituting Eq. (39) into Eqs. (4) we may decompose the total field into the sum of an incoming part and an outgoing part,

$$
\begin{aligned}
& \mathbf{E}_{\text {total }}=\mathbf{E}_{\text {outgoing }}+\mathbf{E}_{\text {incoming }}, \\
& \mathbf{B}_{\text {total }}=\mathbf{B}_{\text {outgoing }}+\mathbf{B}_{\text {incoming }} .
\end{aligned}
$$

The fields $\mathbf{E}_{\text {outgoing }}$ and $\mathbf{B}_{\text {outgoing }}$ contain all the $h_{l}^{\prime \prime \prime}(k r)$ terms, and the fields $\mathbf{E}_{\text {incoming }}$ and $\mathbf{B}_{\text {incoming }}$ contain all the $h_{l}^{(2)}(k r)$ terms. The partial-wave expansion of the outgoing fields is identical to Eqs. (10) except that $a_{l}$ and $b_{l}$ are replaced by

$$
A_{l}=a_{l}-1 / 2, \quad B_{l}=b_{l}-1 / 2,
$$

respectively. Similarly, the partial-wave expansion of the incoming fields is identical to Eqs. (4) except that $j_{l}(k r)$ is replaced by $1 / 2 h_{l}^{(2)}(k r)$.

In a number of respects the incoming and outgoing fields have a pleasing physical interpretation. First, the Debye-series expansion of the incoming and outgoing partial-wave amplitudes is sensible. For the incoming fields, using a shorthand notation, we have

$$
\mathbf{E}_{\text {incoming }} \approx \sum_{l=1}^{k a}\left(-\frac{1}{2}\right)+\sum_{l=k a+1}^{x}\left(-\frac{1}{2}\right) .
$$

In the large-particle limit relation (42) reflects the fact that the incoming beam consists of geometrical light rays 
that will strike the particle (first term) and rays that will miss it (second term). For the outgoing fields we have

$$
E_{\text {outzoing }}=\sum_{l=1}^{k a}\left(\begin{array}{l}
A_{l} \\
B_{l}
\end{array}\right)+\sum_{l=k a+1}^{x}\left(\begin{array}{l}
a_{l} \\
b_{l}
\end{array}\right)+\sum_{l=k a+1}^{x}\left(-\frac{1}{2}\right) \text {. }
$$

Relation (43) reflects the fact that the outgoing radiation consists of geometrical rays that have struck the particle and have been either reflected or transmitted following $p-1$ internal reflections (first term), rays that were incident at the edge of the particle and participated in tunneling reflection or tunneling transmission following $p-1$ internal reflections ${ }^{8,34}$ (second term), and diffracted rays plus the outgoing portion of the original beam that missed the particle (third term). In the large-particle limit the third term in relation (43) may be written as

$$
\begin{aligned}
\mathbf{E}_{\text {outzoing }}^{\text {third } u r m}= & \frac{i E_{0}}{k r} \exp (i k r) \hat{u}_{x} \sum_{l=l_{\text {ax }}}^{x}(l+1 / 2) g_{l}(-1) \\
& \times J_{0}[(l+1 / 2) \theta] \\
\approx & \frac{-i E_{0}}{k r} \exp (i k r) \hat{u}_{x} \int_{k a}^{x} u \mathrm{~d} u \exp \left(-s^{2} u^{2}\right) J_{0}(u \theta) \\
\approx & \frac{-i E_{0}}{k r} \exp (i k r) \hat{u}_{x}\left[\int_{0}^{x} u \mathrm{~d} u \exp \left(-s^{2} u^{2}\right)\right. \\
& \left.\times J_{0}(u \theta)-\int_{0}^{k o} u \mathrm{~d} u \exp \left(-s^{2} u^{2}\right) J_{0}(u \theta)\right]
\end{aligned}
$$

For $w_{0} / a \gg 1$ the first term of the last two lines of Eq. (44) is the undeflected portion of the beam [see relation (28)], and the second term is the circular-aperture Fraunhofer diffraction field of relation (37). For $w_{0} / a<$ $<1$ the last two lines of Eq. (44) contains only the remainder diffraction term of relation (38). The outgoing fields in relation (43) are thus free of the unobservable compensating term that was a necessary part of the scattered fields for $w_{0} / a<1$ in the decomposition of Eqs. (9).

The decomposition of the total fields into a sum of incoming and outgoing waves also provides a sensible description of conservation of energy for scattering by a single particle. Let us define the incoming and outgoing cross sections as

$$
\begin{aligned}
C_{\text {incoming }}= & \frac{-c}{E_{0}^{2}} \int_{0}^{\pi} \sin \theta \mathrm{d} \theta \int_{0}^{2 \pi} \mathrm{d} \phi \\
& \times \operatorname{Re}\left(\mathbf{E}_{\text {incoming }}^{*} \times \mathrm{B}_{\text {incoming }}\right) \\
= & \frac{2 \pi}{k^{2}} \sum_{l=1}^{\infty}(l+1 / 2)|g|^{2}, \\
C_{\text {outgoing }}= & \frac{c}{E_{0}^{2}} \int_{0}^{\pi} \sin \theta \mathrm{d} \theta \int_{0}^{2 \pi} \mathrm{d} \phi \\
& \times \operatorname{Re}\left(\mathbf{E}_{\text {outgoing }}^{*} \times \mathbf{B}_{\text {outgoing }}\right) \\
= & \frac{2 \pi}{k^{2}} \sum_{l=1}^{\infty}(2 l+1)|g l|^{2}\left(\left|A_{l}\right|^{2}+\left|B_{l}\right|^{2}\right),
\end{aligned}
$$

respectively. Since the angle-integrated cross-term Poynting vector vanishes, i.e.,

$$
\begin{aligned}
& \frac{c}{E_{0}^{2}} \int_{0}^{\pi} \sin \theta \mathrm{d} \theta \int_{0}^{2 \pi} \mathrm{d} \phi \\
& \times \operatorname{Re}\left(\mathbf{E}_{\text {incoming }}^{*} \times \mathbf{B}_{\text {outgoing }}+\mathbf{E}_{\text {outgoing }}^{*} \times B_{\text {incoming }}\right)=0,
\end{aligned}
$$

the incoming, outgoing, and absorption cross sections are related by

$$
C_{\text {incoming }}=C_{\text {outgoing }}+C_{\text {absorption }} \text {. }
$$

The outgoing efficiency is defined as

$$
\epsilon_{\text {outgoing }}=\frac{C_{\text {outzoing }}}{C_{\text {incident }}} \text {, }
$$

where $C_{\text {incident }}$ is given by Eqs. (18) and (20).

The outgoing efficiency of Eq. (49) has a problematical behavior for $w_{0} \gg$ a. But a redefinition of $\epsilon_{\text {outgoing }}$ along the lines suggested more than 40 years ago by Brillouin ${ }^{16}$ removes the problem. The problematical behavior of $\epsilon_{\text {outgoing }}$ may be easily demonstrated by the following example. Equation (49) was calculated for a focused on-axis Gaussian beam with $\lambda=0.6328 \mu \mathrm{m}$ and $0.1 \leq w_{0} / a \leq 10$ incident upon a spherical water droplet with $a=50 \mu \mathrm{m}$ and $n=1.333$. The results are shown in Fig. 6. For $w_{0} / a<1$ the outgoing efficiency is 1.0, being due almost entirely to geometrical rays striking the particle surface and deflected by it. Diffraction is negligible. For $w_{0} / a=1$ the beam at the edge of the particle is stronger, diffraction becomes correspondingly stronger, and $\epsilon_{\text {outgoing }}$ increases. This is sensible behavior. But for $w_{0} / a>1$ the outgoing efficiency increases beyond 2.0 , in disagreement with one's intuitive expectation of a maximum value of approximately 2.0 .

The problem is that for $w_{0} / a>1$, in the near-forward direction, the outgoing fields of relation (43) contain not only the diffracted, reflected, and transmitted fields but also the portion of the original beam that missed the particle and now is propagating undeflected toward $z \rightarrow x$ as in Fig. 1. If we wish to describe the efficiency of the interaction of the beam with the particle, this undeflected field must be subtracted from $\mathrm{E}_{\text {outgong. I }}$ I propose to do this in the following way. The far-zone outgoing intensity is constructed from the outgoing fields according to

$$
I_{\text {outgoing }}(\theta, \phi) \hat{u}_{r}=\operatorname{Re}\left(\mathbf{E}_{\text {outgoing }}^{*} \times \mathbf{B}_{\text {outgoing }}\right) .
$$

Since the two largest contributions to the outgoing intensity in the near-forward direction for $w_{0} / a \gg 1$ are

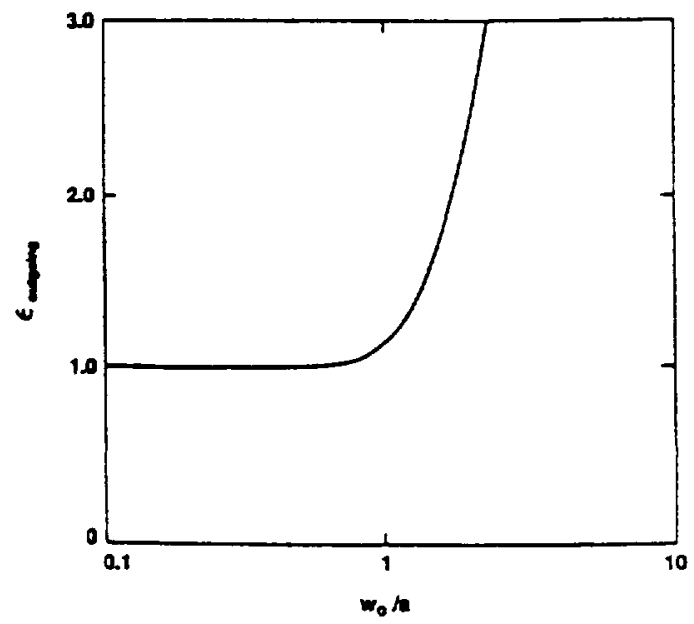

Fig. 6. Outgoing efficiency of Eq. (49) as a function of the half-width of the beam divided by the particle radius. The beam and particle parameters are the same as those in Fig. 3. 


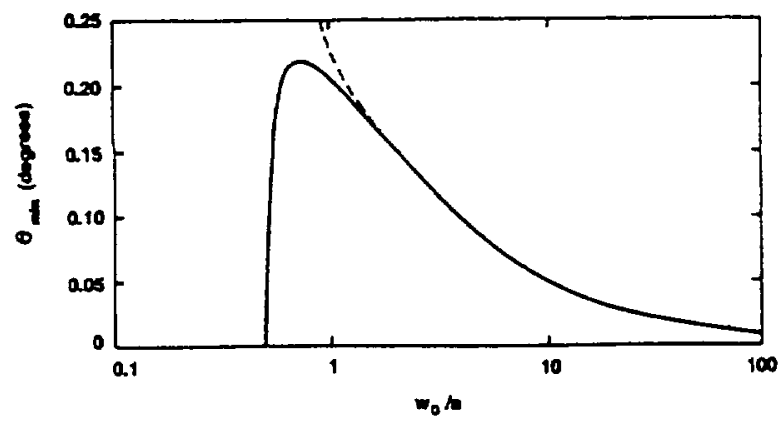

(a)

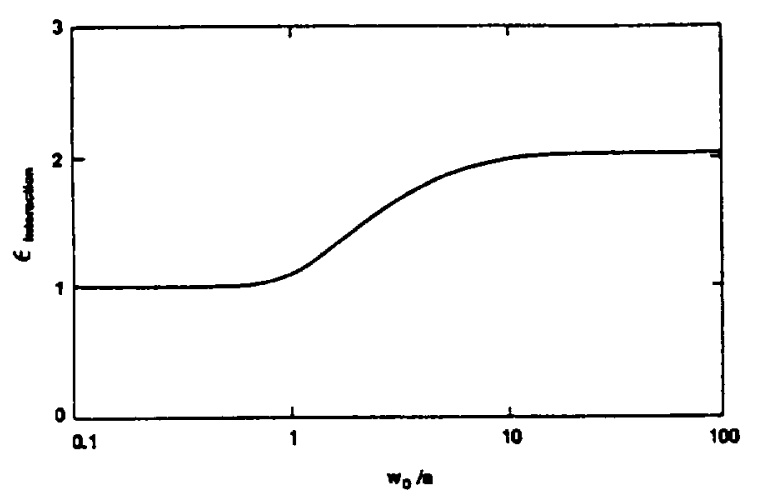

(b)

Fig. 7. (a) Angle of the first minimum of the outgoing intensity of Eq. (50) as a function of the half-width of the beam divided by the particle radius (solid curve). The beam and particle parameters are the same as those in Fig. 3. The dashed curve is the approximation of relation (53). (b) Interaction efficiency of Eq. (52) as a function of the half-width of the beam divided by the particle radius. The beam and particle parameters are the same as those in Fig. 3.

the undeflected original beam of relation (28) and the diffracted wave of relation (37), the first relative minimum of $I_{\text {outgoing }}$ as a function of $\theta$, which we term $\theta_{\min }$, results from the destructive interference of the undeflected beam with the diffracted field and is nearly independent of $\phi$. Following Ref. 16, we claim that any scattering contribution to the outgoing intensity in the angular region $0^{\circ} \leq \theta \leq \theta_{\min }$ and $0 \leq \phi \leq 2 \pi$ cannot be disentangled from the undeflected original beam, since they both decrease as $1 / r$. On the other hand, the outgoing intensity for $\theta_{\min } \leq \theta \leq 180^{\circ}$ is easily recognized as being due to scattering, since it lies beyond the angular interval within which the undeflected beam is confined. When the outgoing intensity is integrated over this range of $\theta$ and $\phi$, the result represents the energy lost from the forward. propagating and spreading Gaussian beam during its interaction with the particle. We define this interaction cross section and efficiency to be

$$
\begin{aligned}
C_{\text {interaction }} & =\frac{c}{E_{0}^{2}} \int_{\theta_{\min }}^{\pi} \sin \theta \mathrm{d} \theta \int_{0}^{2 \pi} \mathrm{d} \phi I_{\text {outgoing }}(\theta, \phi), \\
\epsilon_{\text {interaction }} & =\frac{C_{\text {internction }} .}{C_{\text {incident }}} .
\end{aligned}
$$

This subtraction procedure for the undeflected field was numerically tested in the following way. The outgoing intensity of Eq. (50) was computed for a focused on-axis
Gaussian beam with $\lambda=0.6328 \mu \mathrm{m}$ and $0.1 \leq w_{0} / a \leq$ 100 incident upon a spherical water droplet with $a=$ $50 \mu \mathrm{m}$ and $n=1.333$. The angle $\theta_{\min }$ was numerically determined and is shown as a function of $w_{0} / a$ in Fig. $7(a)$. For $w_{0} / a \gg 1, \theta_{\min }$ may be approximately obtained by locating the angle of maximal destructive interference of the undeflected original beam of relation (28) with the diffracted field of relation (37). The result,

$$
\theta_{\min } \approx \frac{\lambda}{\pi w_{0}}\left\{-\ln \left[\frac{a^{2}}{w_{0}^{2}} \exp \left(-a^{2} / w_{0}^{2}\right)\right]\right\},
$$

is shown as the dashed curve in Fig. $7(a)$. As diffraction weakens for $w_{0} / a<1$, the value of $\theta_{\min }$ is determined by the interference between the original beam and the transmitted field, which is the next-largest contribution to $\mathbf{E}_{\text {outgoing. }}$ For $w_{0} / a \ll 1, \theta_{\min }$ decreases to zero at the size parameters for which the beam field and the transmitted field destructively interfere as in Fig. 7(a). On the other hand, at the size parameters for which the beam field and the transmitted field constructively interfere for $w_{0} / a \ll$ 1 , the value of $\theta_{\min }$ levels out at a small nonzero value.

The interaction efficiency of Eq. (52) was computed with a 7200-point grid for the $\theta$ integration in $C_{\text {interaction }}$. The results are shown in Fig. 7(b). For $w_{0} / a \gg 1$ the interaction efficiency is approximately 2.0. Of this amount, approximately 1.0 is due to scattering and approximately 1.0 is due to diffraction. For $w_{0} / a \approx 1$ diffraction weakens, and the interaction efficiency correspondingly decreases. For $w_{0} / a \ll 1$ virtually no diffraction occurs, and the interaction efficiency is due almost entirely to scattering. Similar results occur for other wavelengths and particle sizes and are consistent with our intuition about diffraction for both wide and narrow beams. I claim that, for Gaussian-beam scattering, the interaction cross section of $\mathrm{Eq}$. (51) represents the best measure of the energy lost by the incident beam resulting from its interaction with a single spherical particle.

This entire development was for an on-axis Gaussian beam. The extinction cross section for an off-axis Gaussian beam has been derived in Ref. 35. Although we have not numerically analyzed this case, the extinction efficiency presumably also behaves in a counterintuitive way for $w_{0} / a<1$, and a corresponding interaction efficiency may also be defined.

\section{APPENDIX A}

Consider diffraction of an incident beam by an aperture in the Fresnel-Kirchhoff approximation ${ }^{36}$

$$
\mathbf{E}^{\mathrm{diff}}(z)=\frac{-i k}{2 \pi z} \int \mathrm{d}^{2} r^{\prime} \mathbf{E}_{\text {incident }}\left(\mathbf{r}^{\prime}\right) A\left(\mathbf{r}^{\prime}\right) \exp \left(-i k \hat{u}_{r} \cdot \boldsymbol{r}^{\prime}\right)
$$

where $\mathbf{r}$ is the vector from the center of the aperture plane to the position of the observer a distance $z$ away, $\mathbf{r}^{\prime}$ is the vector from the center of the aperture plane to any point in the aperture, $\mathbf{E}_{\text {incident }}\left(\mathbf{r}^{\prime}\right)$ is the incident electric field in the aperture plane, and the aperture function $A\left(\mathbf{r}^{\prime}\right)$ is defined by

$$
A\left(\mathbf{r}^{\prime}\right)=\left\{\begin{array}{ll}
1 & \text { inside the aperture } \\
0 & \text { outside the aperture }
\end{array} .\right.
$$


Let the incident beam be a focused on-axis Gaussian beam whose electric field in the aperture plane is

$$
\mathbf{E}_{\text {incident }}\left(\mathbf{r}^{\prime}\right)=E_{0} \exp \left(-r^{\prime 2} / w_{0}^{2}\right) \hat{u}_{x} \exp (i k z)
$$

and let the aperture be the region exterior to a circle of radius $a$. This is appropriate for describing diffraction by a spherical obstacle. If the observer is at the scattering angles $(\theta, \phi), \mathrm{Eq}$. (A1) becomes

$$
\begin{aligned}
\mathbf{E}^{\mathrm{di \pi}}(z)= & \frac{-i k E_{0}}{2 \pi z} \int_{a}^{x} r^{\prime} \mathrm{d} r^{\prime} \int_{0}^{2 \pi} \mathrm{d} \xi \exp \left(-r^{\prime 2} / w_{0}{ }^{2}\right) \\
& \times \exp \left[-i k r^{\prime} \sin \theta \cos (\xi-\phi)\right] \hat{u}_{x} \exp (i k z) \\
= & \frac{-i k E_{0}}{z} \hat{u}_{x} \int_{a}^{\infty} r^{\prime} \mathrm{d} r^{\prime} \exp \left(-r^{\prime 2} / w_{0}{ }^{2}\right) J_{0}\left(k r^{\prime} \sin \theta\right) \\
& \times \exp (i k z) .
\end{aligned}
$$

The strength of the incident electric field at the edge of the aperture is $\exp \left(-a^{2} / w_{0}^{2}\right)$. It falls to $1 / e$ of this value at

$$
r^{\prime}=a+\Delta r^{\prime} \approx a+\frac{w_{0}^{2}}{2 a} .
$$

The value of the last integral of Eq. (A4) is then crudely approximated by

$$
\begin{aligned}
& \int_{a}^{x} r^{\prime} \mathrm{d} r^{\prime} \exp \left(-r^{\prime 2} / w_{0}^{2}\right) J_{0}\left(k r^{\prime} \sin \theta\right) \\
& \approx a\left(\frac{w_{0}^{2}}{2 a}\right) \exp \left(-a^{2} / w_{0}^{2}\right) J_{0}(k a \theta),
\end{aligned}
$$

giving

$$
\mathbf{E}^{\mathrm{diff}}(z)=\frac{-i E_{0}}{k z} \exp (i k z) \frac{k^{2} w_{0}^{2}}{2} \exp \left(-a^{2} / w_{0}^{2}\right) J_{0}(k a \theta) \hat{u}_{x},
$$

in agreement with relation (38).

\section{ACKNOWLEDGMENT}

This research was supported in part by National Aeronautics and Space Administration grant NCC-3-204.

\section{REFERENCES AND NOTES}

1. H. C. van de Hulst, Light Scattering by Small Particles (Dover, New York, 1981), Sec. 2.2.

2. C. F. Bohren and D. R. Huffman, Absorption and Scattering of Light by Small Particles (Wiley, New York, 1983), Sec. 3.4.

3. Ref. 1 , Sec. 18.32 .

4. Ref. 2, Sec. 3.4.1.

5. M. Kerker, The Scattering of Light and Other Electromagnetic Radiation (Academic, New York, 1969), p. 325.

6. Ref. 2, Secs. 4.4.3, 7.1.1, and 7.1.2.

7. Ref 5 , Sec. 4.2.1 a.

8. J Lock and E. A. Hovenac, "Diffraction of a Gaussian beam by a spherical obstacle," Am. J. Phys. 61, 698-707 (1993).

9. L. W. Davis, Theory of electromagnetic beams," Phys. Rev. A 19, 1177-1179 (1979).
10. G. Gouesbet, G. Gréhan, and B. Maheu, "Scattering of a Gaussian beam by a Mie scatter center using a Bromwich formalism," J. Opt. (Paris) 16, 83-93 (1985).

11. J. A. Lock, "Contribution of high-order rainbows to the scattering of a Guassian laser beam by a spherical particle," J. Opt. Soc. Am. A 10, 693-706 (1993).

12. G. Gréhan, B. Maheu, and G. Gouesbet, "Scattering of laser beams by Mie scatter centers: numerical results using a localized approximation," Appl. Opt. 25, 3539-3548 (1986).

13. Ref. 1 , Sec. 9.22 .

14. Ref. 5, Sec. 3.3.2.

15. Ref. 2, Sec. 4.3.3.

16. L. Brillouin, The scattering cross section of spheres for elec tromagnetic waves," J. Appl. Phys. 20, 1110-1125 (1949).

17. H. M. Nussenzveig, "High-frequency scattering by an impenetrable sphere," Ann. Phys. (N.Y.) 34, 23-95 (1965)

18. Ref. 5, Sec. 3.3.3.

19. H. M. Nussenzveig and W. J. Wiscombe, "Efficiency factors in Mie scattering," Phys. Rev. Lett. 45, 1490-1494 (1980).

20. J. A. Lock and L. Yang, "Interference between diffraction and transmission in the Mie extinction efficiency," J. Opt. Soc. Am. A 8, 1132-1134 (1991).

21. P. Chyllek "Partial-wave resonances and the ripple structure in the Mie normalized extinction cross section," J. Opt. Soc. Am. 66, 285-287 (1976)

22. J. P. Barton, D. R. Alexander, and S. A. Schaub, "Internal fields of a spherical particle illuminated by a tightly focused leser beam: focal point positioning effects at resonance," J. Appl. Phys. 65, 2900-2906 (1989).

23. J.-P. Chevaillier, J. Fabre, G. Grêhan, and G. Gouesbet, "Comparison of diffraction theory and generalized LorenzMie theory for a sphere located on the axis of a laser beam," Appl. Opt. 29, 1293-1298 (1990).

24. F. Guilloteau, G. Grèhan, and G. Gouesbet, "Optical levitation experiments to assess the validity of the generalized Lorenz-Mie theory," Appl. Opt. 31, 2942-2951 (1992).

25. J. A Lock, "Improved Gaussian beam scattering algorithm," Appl. Opt. 34, 559-570 (1995).

26. Ref. 1, Sec. 12.32

27. The conversion of a sum over partial waves to an integral over impact parameter in the semiclassical limit is discussed in Ref. 28 below and in K. W. Ford and J. A. Wheeler, "Semiclassical description of scattering," Ann. Phys. (N.Y.) 7, 259-286 (1959); M. V. Berry and K. E. Mount, "Semiclassical approximation in wave mechanics," Rep. Prog. Phys. 35, 315-397 (1972), Sec. 6.

28. H. M. Nussenzveig, "High frequency scattering by a transparent sphere. I. Direct reflection and transmission," J. Math. Phys. 10, 82-124 (1969).

29. J. A. Lock, "Cooperative effects among partial waves in Mie scattering," J. Opt. Soc. Am. A 5, 2032-2044 (1988).

30. A. S. Chaj and H. J. Wertz, The digital computation of the far-field radiation pattern of a truncated Gaussian aperture distribution," IEEE Trans. Antennas Propag. AP-13, 994-995 (1965)

31. G. O. Olaofe, "Diffraction by Gaussian apertures," J. Opt. Soc. Am. 60, 1654-1657 (1970).

32. R. G. Schell and G. Tyras, "Irradiance from an aperture with a truncated-Gaussian field distribution," J. Opt. Soc. Am. 61, 31-35 (1971).

33. G. Arfken, Mathematical Methods for Physicists, 3rd ed. (Academic, New York, 1985), p. 627.

34. H. M. Nussenzveig and W. J. Wiscombe, "Diffraction as tunneling," Phys. Rev. Lett. 59, 1667-1670 (1987).

35. G. Gouesbet, B. Maheu, and G. Grehan, Light scattering from a sphere arbitrarily located in a Gaussian beam, using aromwich formulation," J. Opt Soc. Am. A 9, 1427-1443 (1988).

36. J. W. Goodman, Introduction to Fourier Optics (McGrawHill, New York, 1968), Eq. (4-13). 